- Presents a potentially serious complication arising from the attempted routine extraction of a tooth.

- Describes when removal of a fractured maxillary tuberosity should not be attempted.

\title{
An extraction complicated by lateral and medial pterygoid tethering of a fractured maxillary tuberosity
}

\author{
N. Shah ${ }^{1}$ and J. B. Bridgman ${ }^{2}$
}

We report a case in which the extraction of an upper second molar was complicated by a maxillary tuberosity fracture. Delivery of the tooth and bone fragment under local anaesthesia was unable to be achieved because of pain, brisk bleeding and tethering by the lateral and medial pterygoid muscles. The eventual removal of the fragment under general anaesthetic required the control of haemorrhage deep within the infratemporal fossa. When this complication is recognised by the general dentist the maxillary tuberosity should not be removed and the patient referred to a specialist unit.

\section{CASE REPORT}

A 50-year-old female was referred to our unit by her general dental practitioner after attempting to extract her upper right second molar tooth with forceps. A maxillary tuberosity fracture occurred and the decision was made to remove the tooth and the tuberosity as the segment appeared small. However both the dentist and later a surgical dentist were unable to dissect the soft tissues free of the bony fragment due to brisk haemorrhage, inadequate analgesia and tenacious soft tissue tethering. The area of bleeding was arrested with an absorbable haemostatic agent and sutures. The patient was then referred on to the maxillofacial department of Queen Victoria Hospital, East Grinstead, UK.

On presentation to our unit there was no bleeding from the site but the area was

\footnotetext{
${ }^{1 *}$ Author position, Maxillofacial Unit, Queen Victoria Hospital, East Grinstead, West Sussex, RH19 3DZ:

${ }^{2}$ Author position, address

*Correspondence to: Dr Noman Shah

Email:maxfax73@yahoo.co.uk
}

\section{Refereed Paper}

Received 28.06.04; Accepted 15.09.04

doi: 10.1038/sj.bdj.4812302

๑ British Dental Journal 2005; 198: 543-544 very tender to examination from the two previous surgical interventions. A panoramic radiograph revealed the fractured segment of bone and depicted an enlarged maxillary sinus without any other notable risk factors of a tuberosity fracture. The patient understandably requested a general anaesthetic for any further attempt to resolve the problem. Under general anaesthetic the tooth and attached bony fragment complex was found to be freely mobile but felt to be tethered by a deep, strong and elastic structure. Exploration revealed the bone to have a strong muscle attachment which could not be released by sub-periosteal dissection because of uncontrollable deep bleeding and access limitations, and so it was freed with surgical scissors. The brisk bleeding in the region appeared to be from a small artery supplying the muscle which had retracted into the infratemporal fossa. There was also a general muscle bed ooze. A stainless steel clip was used to seal the artery and the application of bipolar diathermy controlled the muscle bleeding. An oro-antral communication large enough to place a finger tip into could be felt on the inferior posterior wall of the maxillary sinus. Resorbable horizontal mattress sutures were used to close the wound primarily. Post operatively, a five day course of Co-Amoxyclav and a Chlorohexidiene mouthwash were prescribed together with adequate pain killers. In addition to the usual post extraction instructions the patient was advised to avoid blowing her nose for two weeks to help prevent an oro-antral fistula from developing. The patient had an uneventful recovery and has a full comfortable range of mandibular movements.

Examination of the removed specimen, (Fig. 1), proved the attached bone to include the maxillary tuberosity, the pyramidal process of the palatine bone and a small portion of the lateral pterygoid plate. The main tethering muscle was lateral pterygoid but also some fibres from medial pterygoid were noted.

\section{DISCUSSION}

The lateral and medial pterygoid muscles lie within the infra-temporal fossa which is a space located deep to the ramus of the mandible and posterior to the maxilla. The lateral pterygoid muscle largely arises from the lateral surface of the lateral pterygoid plate and the medial pterygoid muscle arises mainly from the medial side 


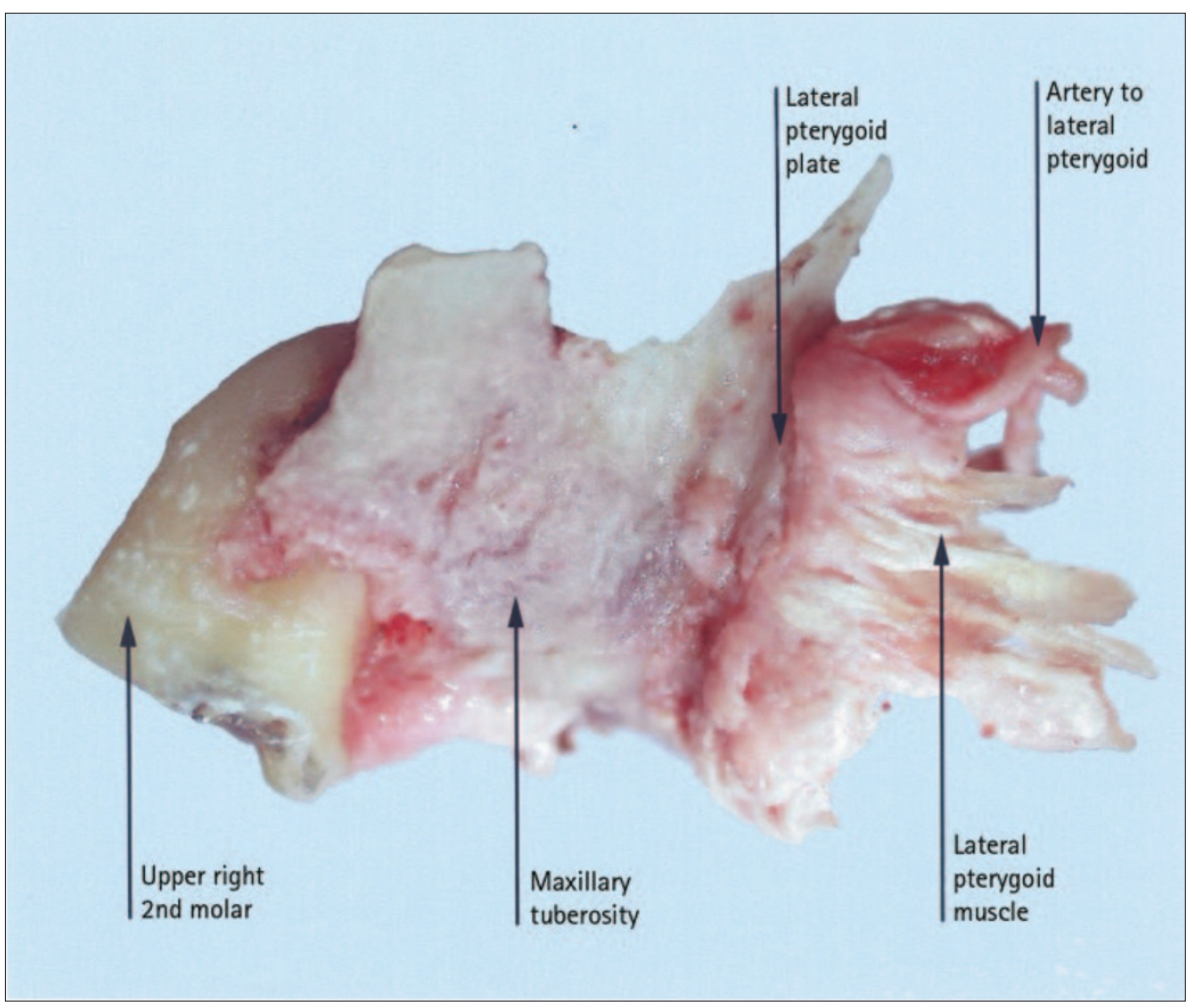

Fig. 1 The lateral view of the removed tooth and bone complex demonstrating the attachment of the lateral pterygoid muscle

of the lateral pterygoid plate. A smaller superficial head of medial pterygoid also arises from the maxillary tuberosity and the neighbouring part of the pyramidal process of the palatine bone immediately above the tuberosity. Concentrated around and within the lateral pterygoid muscle lies a venous network, the pterygoid plexus of veins. ${ }^{1}$

Our examinations of dry skulls and preserved dissections available to us through the medical school at Guy's Campus, London, revealed that the bony architecture and the muscle attachments around the maxillary tuberosity and lateral pterygoid plate can be highly variable. It appears from examination of the removed specimen in this case that there was a relatively short distance between the maxillary tuberosity and the start of the lateral pterygoid plate which may have predisposed the patient to this complication.

It is commonly advised that if a decision is made to remove the fractured tuberosity the soft tissue attachments should be carefully dissected off the hard tissue fragment. Specific muscle tethering of a maxillary tuberosity fracture, is not discussed in oral and maxillofacial textbooks available to us ${ }^{2-6}$ or in larger review papers $^{7,8}$ on the topic. Serious complications of maxillary tuberosity fracture have ligaments. attempting to retain the bone. ${ }^{2,7}$ One reason that is frequently stated as an indication for conserving the fractured tuberosity is that removal makes later denture reconstruction difficult, although this is questioned. ${ }^{7}$ If the bone of the tuberosity is going to be attempted to be retained it is treated with the same surgical principles as other dentoalveolar fractures. The tooth can be dissected away from the bony fragment, and provided there is sufficient periosteal attachment, the segment can be held by appropriate suturing to allow bone union to take place. Alternatively, the tooth and bone complex segment can be immobilised, allowed to heal and the tooth or teeth extracted surgically at a later time. For this later technique the state of the tooth, its dental pulp and its place in occlusion also need to be managed. In all cases attention must be given to any communication established with the maxillary sinus. ${ }^{2,5-7}$

This complication occurred as a result of a routine dental procedure under local anaesthetic and was correctly referred to a specialist unit. Once the nature of the problem had been elucidated, the management of this case under general anaesthetic in the operating theatre setting was relatively straightforward. However, in the outpatient setting with the patient conscious, it would have been almost impossible and potentially dangerous.

The authors wish to thank Professor Susan Standring of Guy's Medical School, London for her help in the anatomical inquiry for this report and to our consultants Mr A. Brown and Mr K. Lavery for their helpful comments in the preparation of this report. collapsing the opening of the eustac mandibular movements from the disruption of the pterygoid muscles and

A maxillary tuberosity is thought to be more predisposed to fracture if the maxillary sinus has enlarged between the teeth and into the tuberosity so creating thin bony walls. Dental anomalies of the maxillary molars may also be contributory including; tooth fusion, tooth isolation, over eruption, ankylosis, hypercementosis, chronic periapical infection and roots which are widely divergent. If it is thought that there is a high risk of a maxillary tuberosity fracture occurring then a surgical extraction of the molar is generally recommended. $2,7,10$

Two philosophies of management of a fractured maxillary tuberosity are commonly put forward. If the bone fragment is large it may be attempted to be salvaged $^{5,6}$ but immediate removal may be a better choice because of the difficulty in
1. Berkovitz B K B, Moxham B J. A textbook of head and neck anatomy. pp 174-176. Barcelona: Wolfe Medical Publications Ltd, 1988.

2. Howe G L. Minor oral surgery. $3^{\text {rd }}$ ed. pp 118-121. London: Wright, 1985.

3. Thoma K H. Oral surgery. $3^{\text {rd }}$ ed. pp 208. London: Henry Kimpton, 1958.

4. Robinson P D. Tooth extraction: A practical guide. pp 104-105. Oxford: Wright, 2000.

5. Laskin D M. Oral and maxillofacial surgery. pp 32-33. St Louis: The C.V. Mosby Company, 1985.

6. Peterson LJ . Contemporary oral and maxillofacial surgery. pp 270-271. St Louis: The C.V. Mosby Company, 1988.

7. Norman J E deB, Cannon P D. Fracture of the maxillary tuberosity. Oral Surg., Oral Med \& Oral Path.

8. Cohen L. Fractures of the maxillary tuberosity occurring during tooth extraction. Oral Surg, Oral

9. Coleman F. Extraction of teeth. pp 76. London: H.K. Lewis, 1908. In Cattlin W. A paper on the form and size of the adult antrum and on the diagnosis and treatment of inflammation of the lining membrane. Tr Odont Soc 1858; 2: 31-46.

10. Altonen $\mathrm{M}$, Rantanen A V. Factors increasing the risk of fracture of the maxillary tuberosity during tooth extraction. Pro Finn Den Soc 1976: 72: 163-169. 1967; 24: 459-467. Med \& Oral Path 1960; 13: 409-411. 